PAPER

\title{
The impossibility of informed consent?
}

\author{
Kenneth Boyd
}

\section{Correspondence to \\ Professor Kenneth Boyd, Biomedical Teaching \\ Organisation, Edinburgh University, Edinburgh, Scotland EH89AG, UK; \\ K.Boyd@ed.ac.uk}

Received 1 September 2014 Revised 26 September 2014 Accepted 23 October 2014

\begin{abstract}
The problematic nature of informed consent to medical treatment and research, and its relation to autonomy, trust and clinical practice, has been addressed on many occasions and from a variety of ethical perspectives in the pages of the Journal of Medical Ethics. This paper gives an account of how discussion of these issues has developed and changed, by describing a number of significant contributions to these debates which provide examples of 'doing good medical ethics' over the 40 years of the Journal's publication.
\end{abstract}

\section{THE IMPOSSIBILITY OF INFORMED CONSENT?}

Awareness that informed consent is problematic, in theory and in practice, was already evident in the earliest editions of the Journal of Medical Ethics. 'Is the doctor to warn the patient of every conceivable complication and give her the statistical data? Is there such a thing as 'truly informed' consent?' asked an editorial ${ }^{1}$ in September 1975, discussing a 'woman's right to choose' abortion. In the same issue, serious doubts about informed consent to participation in clinical research were raised in a paper by the British clinical pharmacologist JC Garnham. ${ }^{2}$ This presented evidence that only 'medically trained people', and sometimes not even all of them, when recruited as subjects of nontherapeutic clinical research, demonstrated sufficient understanding of the implications of their voluntary consent for it to be meaningfully considered 'informed'. In the research context therefore, the paper concluded, 'informed consent' had 'little or no meaning'; and it went on to recommend that any request for consent to participation in clinical research should be preceded by information provided by the investigators, and by the registration of medical agreement to participation in the study by the potential subject's own or nominated doctor.

Garnham's paper further recommended that in order to prevent conflicts of interest, research ethics committees should be 'appointed on a geographical basis rather than by institutions'. A decade or so later a national system of such independent committees was set up in the UK, and their role in protecting the interests of research subjects perhaps partly superseded that envisaged by Garnham for the subject's own or nominated doctor. Many other aspects of informed consent however remained and remain contested philosophically and practically, and were to be addressed in the pages of the Journal from a considerable variety of different approaches. The following represent some significant contributions to these debates which provide examples of 'doing good medical ethics' over the 40 years of the Journal's publication.

\section{Surveying the scene: the law and ethics of} informed consent to treatment and research

Two early and wide-ranging contributions on medicolegal and ethical aspects of informed consent were made in papers by judge Kirby ${ }^{3}$ of Australia in 1983 and paediatrician Silverman ${ }^{4}$ of the USA in 1989. Both agreed that a paternalist or beneficencebased approach, in which the doctor recommends a course of treatment to which the patient either consents or refuses, had properly been superseded by an approach based on respect for the autonomy or right to self-determination of the individual patient. Having spelt out the necessity for this latter approach in terms of the law of trespass and of negligence, Kirby admitted that informed consent was 'hard to define' and 'simply an ideal to which daily practice can only aim': it was 'impossible for the healthcare professional to impart... every facet of his knowledge and expertise involved in the decision... in the space of a 30-min consultation' to patients who 'vary enormously in their interest in and capacity to absorb information about medical procedures'. The 'degree of detail and information' to be provided nevertheless had now, in the view of the courts, to be determined not by 'the sole judgement of the medical profession itself' but by 'the patient's need to know and respect for the patient's autonomy'. Ultimately however, and for most doctors reassuringly, Kirby concluded, "The fact that the patient gave an informed consent usually will not prevent him from suing; a warm relationship with a competent and caring physician usually will.'

Silverman's less reassuring paper, entitled 'The myth of informed consent: in daily practice and in clinical trials', was concerned to reconcile the standards and practice of informed consent in clinical practice and clinical research, particularly in the USA. While informed consent ('a modern American invention', first enunciated in a legal case in 1957) required doctors to 'disclose risks and alternatives of treatment' as well as the treatment's 'nature and consequences', there was little evidence, from studies of clinical practice, of doctors being aware that 'the consent-seeking routine was intended to allow patients to make a choice or state a preference about the treatment on offer': doctors still 'felt duty-bound to provide patients with an unequivocal recommendation for action' and thereby to 'minimise the uncertainties about 'best' treatment'. In clinical trials, by contrast, doctors seeking informed consent were now required to be explicit about the uncertainties which justified the
To cite: Boyd K. J Med

44 
need for a trial, but again there was little empirical evidence that this 'ritual' provided research subjects with either any additional protection from the risks of participation, or any increased respect for their autonomy: there was in fact considerable evidence that the majority of participants did not 'fully understand the implications of a formal clinical trial'. It was possible, moreover (a point also noted by Kirby), that the consent process might act as 'an effective social filter', selecting out 'for refusal those on the upper rungs of the social ladder' who were 'most likely to understand what is requested in the consent ritual', leaving among those consenting 'a disproportionate representation' of the 'socially disadvantaged'. This 'apparently irresolvable conflict between the ethical requirement that a patient must always be offered the best treatment known, and the equivocal choice in a comparative trial', Silverman argued, failed to 'satisfy the competing moral imperatives' of respect for autonomy, beneficence and justice. There was however no currently obvious way to 'conserve the spirit of informed consent without snuffing out the flame of responsible clinical study': only by 'experiment with various discretionary approaches" might it become possible to 'strike a balance among the competing interests'.

\section{Fresh insights from psychiatry and philosophy}

Despite Silverman's hopes for reconciliation between the standards for consent to treatment and for consent to research, this issue also was to remain and remains ethically contested. With respect to consent to treatment however, two further papers from the 1980s suggested different ways in which legal requirements might be reconciled with ethical principles and good medical practice. A 1987 paper by Dyer and Bloch, ${ }^{5}$ from the USA. and England, offered insights from their own psychiatric specialty, while one of 1989 by surgeon and ethicist Gillett ${ }^{6}$ from New Zealand suggested a virtue ethics approach to the issue.

Writing against the background of recent legislative and legal developments in England (the Mental Health Act 1983 and the 1984 Sidaway case) Dyer and Bloch observed that while medical paternalism was no longer ethically acceptable, and it was necessary to respect the autonomy of a patient as a person with human rights, this could be very difficult if the autonomy of a patient was limited, for example by an acute psychotic condition or severe dementia. Deciding that they lacked capacity and needed to have their interests represented by a guardian or proxy on the other hand did not restore these patients' autonomy, and at worst could be an impersonal form of official paternalism. This dilemma or conflict between the principle of autonomy and the principle of paternalistic beneficence in relation to informed consent could be resolved however by introducing an ethical ideal even more basic than either-the fiduciary principle of partnership, in which the doctor decides not for but 'with the patient, a process dependent on the development of the patient's trust' and one which while 'requiring mutual trust... particularly calls on the doctor to be trustworthy'. This ideal was exemplified in psychotherapy, for which a trusting relationship was essential if the goal of both partners-'to understand and clarify the motives of the patient'-was to be achieved: this however necessarily took time, meaning that informed consent 'occurs not once, but repeatedly... as a continuing process'.

This (later widely accepted) view of informed consent not as an act but as a process was shared by Gillett in his 1989 paper. This enquired (again against the background of the Sidaway decision) whether, especially in a legally disputed case, it was possible to decide in retrospect if consent had been informed by what was 'of material relevance to a prudent or reasonable patient'. Given on the one hand the need for the consent process to be sufficiently informal 'to allow free communication' (often 'on a ward round or in a bedside conversation' with 'no detailed record of the conversation' in 'the medical notes') and given on the other hand the 'confounding effects of stress and illness' and the fallibility of memory, it could subsequently be 'difficult, if not impossible to reconstruct exactly what happened on an occasion where consent has become an adversarial issue'. In such circumstances, Gillett believed, it was helpful to consider the philosopher Peter Winch's 'analysis of retrospective moral evaluation'. Winch had argued that 'when the facts are unclear it may be that the best guide we have to the rightness of what was done is what a morally endorsable agent actually did as a participant in the situation'. In other words, was the doctor concerned 'a person of the requisite moral character who acted with an informed grasp of the situation'? Ultimately the only way for a moral spectator scrutinising the doctor's actions to answer these questions, was by trying to discover, possibly from others among his patients in similar circumstances, 'the known practice of the doctor concerned, as materially relevant to the present decision'. If enquiries into the doctor's 'track record' satisfied the observer that the doctor was 'a competent moral judge' who 'acted in accordance with adequate principles', then it should be accepted that his actions were 'likely to have been the right thing to do or perhaps $a$ right thing to do in the situation'.

\section{Informed consent requirements in changing contexts}

Several papers from the late 1990s and early 2000s illustrate ethical arguments about how changing contexts and new developments in clinical practice and healthcare research may require different standards for informed consent. Some of these papers related to contemporary events such as the organ retention scandals in and around $2000,{ }^{7}$ or to the acceptability of 'broad consent' for future research from the new biobanks. ${ }^{8}$ Others however were concerned with and developed themes discussed earlier in the pages of the Journal.

A 1998 paper by ethicist Hansson ${ }^{9}$ from Sweden and one of 2002 by health services researchers Cassell and Young ${ }^{10}$ from England, for example, returned to the issues discussed earlier by Silverman concerning different standards for clinical practice and for medical research, but also drew attention to a further relevant distinction between clinical trials and epidemiological or health services research. Noting a 'tendency today for the rule (of informed consent) to be applied too rigidly and with too little sensitivity to the values that are at stake in connection with different kinds of research protocols', Hansson proposed 'a model for balancing the quality of consent against other variables that are ethically important in different contexts'. Hansson's model balanced the quality of consent 'against the time that is available for the communication process and against the values that are at stake for the individual most directly concerned': where values vital for the person concerned ('integrity, health and well-being') were involved and ample time available (as in clinical trials), extensive informed consent procedures were required, but where time was short (as in medical emergencies) less elaborate procedures providing more limited information were sufficient; and in cases where there was no serious threat to the individual's integrity or confidentiality for example (as in epidemiological research seeking information that 'is not judged to be sensitive or socially stigmatising for any 
individual'), simply making available information relevant to enable a right to refusal might be sufficient.

While agreeing that applying the same standards of informed consent in all healthcare and research contexts was ethically inappropriate, Cassell and Young criticised the single standard from a different angle with particular relevance to health services research in the National Health Service. Such research, into the organisation and delivery of healthcare, they argued, was ethically as well as practically necessary in order to 'equalise opportunities for health'. When time-consuming standards of informed consent designed for clinical trials were insisted upon by ethics committees, this could obstruct healthcare research by discouraging participation, allowing patients to opt out, and making health service research possible only with unrepresentative samples. This was especially inappropriate in the National Health Service, of which by its unique nature all UK citizens essentially were not consumers or patients but members, and as members had a common obligation to protect the equal rights of all other members-rights which were not respected if the organisation and delivery of healthcare, and organisational change in the health service, were not properly evaluated by health services research.

\section{Calling autonomy to account}

One of the most common assumptions about informed consent - that its ethical basis is respect for autonomy or the selfdetermination of the patient-was radically challenged in a 2003 paper by the Kantian philosopher O'Neill ${ }^{11}$ from the UK, entitled 'Some limits of informed consent'. Among these limits, O'Neill observed, was that only competent patients could give informed consent; and even among them, this was practically difficult or impossible for those rendered vulnerable by illness or dependency, for relatives of patients who shared information about them with their doctors, or for individuals who would be affected by public health policies. Equally significant, was what O'Neill called the 'opacity' and lack of 'transitivity' of informed consent: a patient giving consent to a medical procedure might be unaware of all the possibilities that it might entail, including those to which they might not have consented had they known of them, yet spelling out in detail every last implication on a complex consent form could be confusing and counterproductive. Part of the problem behind all this, O'Neill argued, was the 'endlessly repeated but deeply obscure' claim that informed consent was 'the key to respecting patient autonomy'. This claim surely could not be correct since the choices protected by informed consent included those that were autonomous and 'choices that are timid, conventional, and lacking in individual autonomy'. The real ethical importance of informed consent rather, O'Neill continued, was 'to provide reasonable reassurance that a patient (research subject, tissue donor) has not been deceived or coerced', and this could be assured in practice by giving them a limited amount of accurate and relevant information and providing user-friendly ways for them to extend this amount (thereby checking that they are not deceived) as well as easy ways of rescinding consent (thereby checking that they are not coerced)'.

O'Neill's arguments were to be influential: set out at greater length in her 2001 Gifford Lectures, they included the claim that informed consent was 'generally important (inter alia) because it can make a distinctive contribution to the restoration of trust'. ${ }^{12}$ Arguments by O'Neill and others in support of this claim however have more recently been challenged in a 2014 paper by ethicist Eyal $^{13}$ from the USA. The argument that informed consent promotes trust in medical practice, Eyal argues, while initially appealing, does not necessarily hold up for a variety of reasons: trust in doctors, for example, is not invariably desirable, especially if it is excessive, while on the other hand trust may actually be diminished, if overemphasising a need for trust or providing too much information makes patients, reasonably or unreasonably, suspicious. There may moreover be some circumstances in which patients have greater trust in doctors whom they believe in the future will act in their best interests rather than abandoning them to an informed refusal they might later live or not live to regret. Promoting trust, Eyal concludes, cannot be seen as the only reason for informed consent procedures which need to be supported by other reasons.

Two final papers from the Journal of Medical Ethics return to considerations of autonomy and in that context argue for further qualifications of, or constraints on informed consent. In their 1997 paper $^{14}$ entitled 'Should informed consent be based on rational beliefs?', ${ }^{13}$ physician and ethicist J Savulescu from the UK and philosopher Momeyer from the USA argue that being autonomous 'may not require that one's choices and actions are rational' but 'it does require that one's beliefs which ground those choices are rational.' Being autonomous 'involves freely and actively making one's own evaluative choices about how one's life should go', but 'we cannot form an idea of what we want without knowing what the options on offer are like'. Applying these considerations to refusal of life-prolonging blood transfusions by Jehovah's Witnesses, Savulescu and Momeyer argue that the beliefs on which this refusal is based are 'irrational on at least two counts: their particular beliefs are not responsive to evidence nor are their interpretations of Biblical text consistent' either with other Biblical texts contradicting their beliefs or with 'the vast majority ... in the Judeo-Christian tradition”. While a Jehovah's Witness could still make an autonomous choice to refuse blood even if they accepted this irrationality and adopted 'relevant informed, rational beliefs', nevertheless as long as their beliefs remained irrational their autonomy would be in question. Savulescu and Momeyer emphasise however that they are employing the example of Jehovah's Witnesses, not to coerce Jehovah's Witness in particular, but simply to illustrate the more general argument that in seeking informed consent, doctors as also educators have a responsibility, if their patients hold irrational beliefs, not simply to inform and then 'abandon' them, but also to help 'patients to deliberate more effectively'.

Similar considerations have been addressed more recently in a 2014 paper by ethicist Levy ${ }^{15}$ from Australia, entitled 'Forced to be free? Increasing patient autonomy by constraining it.' Citing a variety of empirical psychological studies illustrating the 'fallibilities of human reasoning' (including 'myopia for the future', 'motivated reasoning' and 'biases' in 'assessing probabilities... exacerbated... under cognitive load'), Levy argues that 'Human beings are, under a variety of conditions, systematically bad reasoners, and many of their reasoning faults can be expected to affect the kind of judgements that they make when they are called upon to give informed consent'. Because such fallibilities 'threaten to undermine autonomy, but the purpose of securing informed consent is to protect and promote autonomy', he continues, 'we have good reason to redesign the informed consent procedure in ways that help to avoid these fallibilities, even if the redesign reduces the scope for individual decision making in the procedure'. There might, Levy concludes, be 'a case for introducing informed consent specialists', who would 'have the job of detecting cognitive illusions in patients and informing them if they are likely to be at work in their decision-making'. 
While Levy's suggestion of these additional 'strangers at the bedside' seems a possibility less likely to be generally favoured than Savulescu and Momeyer's variation on the traditional educational role of doctors, these early 21 st century papers suggest in medical ethics a possible rebalancing (as suggested by Hansson) of beneficence over autonomy. In a commentary on Levy's paper the US ethicist Caplan ${ }^{16}$ remarks 'What we need in healthcare is a bit more medical paternalism'. If that is going to be the trend, it will be important to weigh in the balance also from the 1970s and 1980s, on the one hand the concerns of Garnham and Silverman about consent to clinical trials, and on the other, in relation to clinical practice, the insights of Dyer and Bloch about the fiduciary principle of partnership, and of Gillett about virtue ethics.

\section{Competing interests None.}

Provenance and peer review Commissioned; internally peer reviewed.

\section{REFERENCES}

1 Editorial: The abortion issue. J Med Ethics 1975;l:109-10.
2 Garnham JC. Some observations on informed consent in non-therapeutic research. J Med Ethics 1975;|:138-45.

3 Kirby MD. Informed consent: what does it mean? J Med Ethics 1983;9:69-75.

4 Silverman WA. The myth of informed consent: in daily practice and in clinical trials. J Med Ethics 1989;15:6-11.

5 Dyer AR, Bloch S. Informed consent and the psychiatric patient. J Med Ethics 1987;13:12-16.

6 Gillett GR. Informed consent and moral integrity. J Med Ethics 1989;15:117-23.

7 English V, Sommerville A. Presumed consent for transplantation: a dead issue after Alder Hey? J Med Ethics 2003;29:147-52.

8 Hofmann B. Broadening consent-and diluting ethics? J Med Ethics 2009;35:125-9.

9 Hansson MO. Balancing the quality of consent. J Med Ethics 1998;24:182-7.

10 Cassell J, Young A. Why we should not seek individual informed consent for participation in health services research. J Med Ethics 2002;28:313-17.

11 O'Neill O. Some limits of informed consent. J Med Ethics 2003;29:4-7.

12 O'Neill O. Autonomy and trust in bioethics. Cambridge: Cambridge University Press, 2002.

13 Eyal N. Using informed consent to save trust. J Med Ethics 2014;40:437-44.

14 Savulescu J, Momeyer RW. Should informed consent be based on rational beliefs? J Med Ethics 1997;23:282-8.

15 Levy N. Forced to be free? Increasing patient autonomy by constraining it. J Med Ethics 2014;40:293-300.

16 Caplan AL. Why autonomy needs help. J Med Ethics 2014;40:301-2. 